
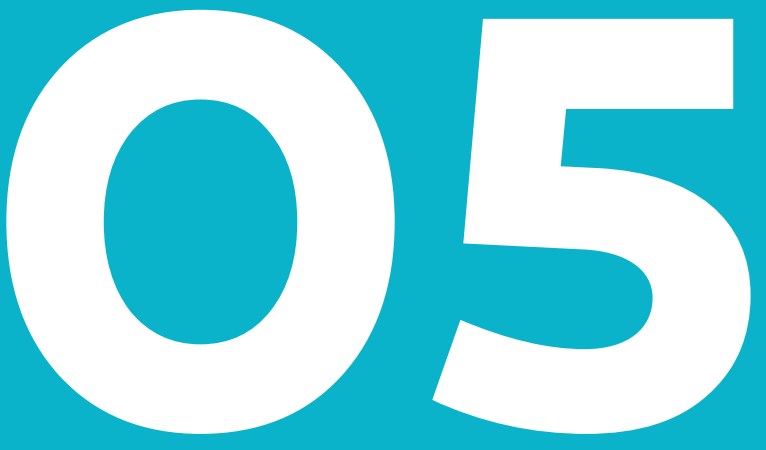

\title{
Estimación de la elasticidad de la demanda de banano fresco en Estados Unidos
}

Estimation of the United States demand's elasticity for fresh bananas

Fecha de recepción: 09/03/2020

Fecha de aprobación: 18/07/2020 


\section{Resumen}

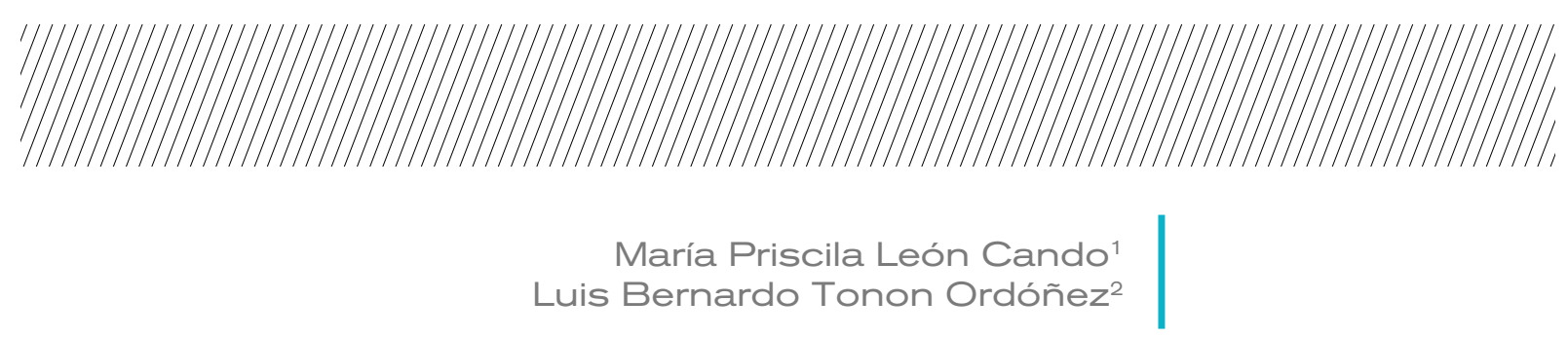

Debido a la importancia del banano en la economía ecuatoriana, es imprescindible analizar la demanda del principal mercado de banano fresco del mundo, Estados Unidos, así como estimar sus elasticidades, priscilaleon@es.uazuay.edu.ec precio y renta de la demanda; $y$, analizar la relación del banano con otras frutas en este mercado. Para la estimación, se utilizó el método de Mínimos Cuadrados Ordinarios. Se determinó que, en este mer-

Banano, Estados Unidos, Estimación demanda, Elasticidad precio,

Elasticidad renta.

cado con potencial de crecimiento, el banano es un bien inelástico y normal. El periodo analizado fue de 2001 a 2016.

Palabras clave: 


\section{Abstract}

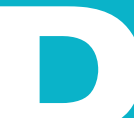

ue to the importance of banana in the Ecuadorian economy, is essential to analyze the demand of the world's principal fresh banana market, United States, as well as estimate its price and income elasticities, and analyze the relationship of bananas with other fruits in this market.

The Ordinary Minimum Squares Method was used for the estimation. It was determinated that in this market with potencial growth, bananas are inelastic, and considered a normal good. The period analyzed was from 2001 to 2016.

Keywords:

Banana, United States, Demand estimation, Price Elasticity, Income Elasticity
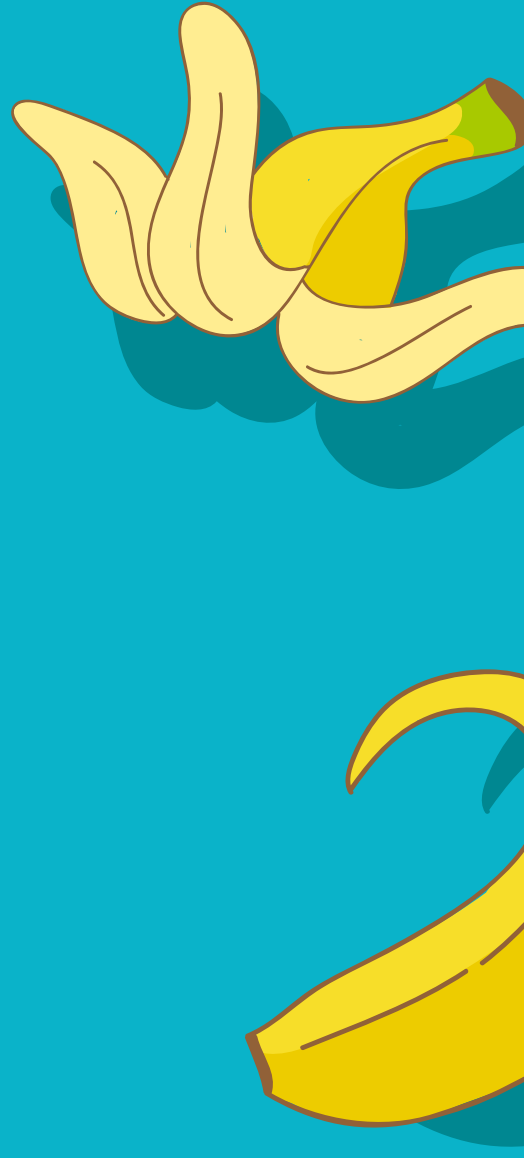

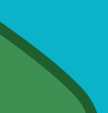


nano fresco del mundo. Con este fin, la presente investigación buscará estimar la demanda interna de banano fresco en estadounidense, para analizar sus elasticidades en el periodo 2001 - 2016.

Para comenzar, deben conocerse ciertos detalles del mercado americano. En primer lugar, en Estados Unidos, la nutrición es un tema de gran importancia para el gobierno, quien busca evitar enfermedades relacionadas como la obesidad, la diabetes, etc.; por lo que a fin de mejorar la educación nutricional y lograr que los ciudadanos consuman una dieta más sana, con ayuda de varias organizaciones, ha formado programas y guías nutricionales donde las frutas son parte importante de la dieta recomendada, pues, existe una correlación positiva entre la información nutricional que reciben los consumidores y la calidad de la dieta de su dieta (Zeballos \& Anekwe, 2018). Tras analizar que ha existido una tendencia creciente del consumo de frutas que, a pesar de los esfuerzos del gobierno no alcanza al consumo óptimo recomendado, se evidencia que este mercado tiene potencial de crecimiento.

Según estudios realizados por Produce Market Guide de The Packer, un periódico que desde 1983 cubre la industria de productos frescos en Estados Unidos, "la popularidad del banano se extiende a todos los grupos demográficos del país" (The Packer, 2013) de diversas edades, razas y niveles de ingreso. En cuanto a preferencia por parte de los consumidores, la misma fuente sostiene que históricamente el banano ha sido la fruta preferida, seguida de la manzana. En 2013, el 84\% de los consumidores dijeron comprar banano; sin embargo, en 2016, por primera vez en más de una década, el banano tomó el segundo puesto de popularidad con $82 \%$ de los consumidores, prefiriendo esta fruta; mientras que, la manzana fue preferida por el 83\% (The Packer, 2016).

Por otra parte, al analizar el consumo per cápita de frutas en Estados Unidos, se puede destacar que, según datos del United States Department of Agriculture (USDA, 2017), en 2015, la principal fruta consumida en sus diversas formas (fresca, enlatada, congelada, seca y en jugo) es la manzana, seguida por la naranja y, en tercer lugar, el banano. Sin embargo, debe diferenciarse que este estudio analiza la fruta fresca $y$, en este caso, el banano esla principal fruta fresca consumida, seguida de la manzana bien de producción nacional. En el periodo 2001-2015, el consumo promedio de banano fue de $4.79 \mathrm{~kg}$ per cápita anuales y, 
de manzana, fue de 4.68; mientras que, de las demás frutas se encontró por debajo de los $2 \mathrm{~kg}$ promedio (USDA, 2017).

En efecto, el banano y la manzana abarcan más del $40 \%$ del consumo de frutas frescas consumida por los americanos (USDA, 2017). Además, al analizar la variación del consumo de la manzana y el banano, este indica que pudieran ser frutas sustitutas entre sí, ya que presentan un comportamiento inverso, en la mayoría de años estudiados, así como que el precio de la manzana presentó una inflación mucho mayor al del banano.

En lo referente al ingreso de los consumidores americanos, según datos del Bureau of Economic Analysis (2018) y el United States Census Bureau (2011) (2017), ha tenido una tendencia creciente entre 2001 y 2016, con una tasa de crecimiento promedio del 3\%; sin embargo, este se ha visto afectado por sucesos de la economía global, pues, presenta un comportamiento similar al del PIB corriente del país, existiendo decrecimientos en épocas de crisis y crecimientos nulos en periodos como el año 2008, tras el inicio de la crisis financiera.

Finalmente, los datos indican que los estadounidenses consumen en promedio el $95 \%$ de su renta y ahorran el $5 \%$ restante (BEA, 2018); además, es importante mencionar que de la renta disponible personal en promedio entre 2001 y 2014 , el $9.6 \%$ fue destinado al gasto total en alimentos, destacando que el $5.6 \%$ fue gasto en alimentos para el hogar; es decir, los comprados en supermercados u otro tipo de tiendas minoristas (USDA, 2016). Estas características presentadas permitirán comprender mejor el comportamiento de un consumidor promedio americano, al estimar su demanda interna de banano fresco.

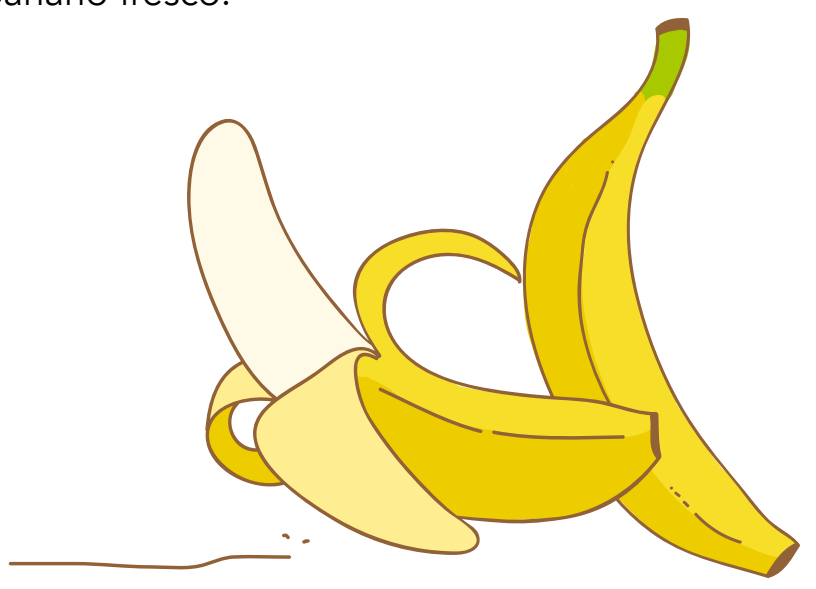




\section{Marco teórico}

Históricamente se define al mercado como aquel lugar donde confluyen oferentes y demandantes de un bien o servicio para realizar sus transacciones. Estos participantes forman las dos fuerzas del mercado conocidas como oferta y demanda, cuyo proceso de convergencia, como afirmaba Adam Smith (1776), era guiado por una mano invisible; por lo tanto, el mercado puede percibirse como una red de productores, usuarios, mayoristas e intermediarios que compran y venden en él (Parkin, 2009).

Con el fin de estudiar el funcionamiento de un mercado, es importante analizar el comportamiento de sus actores (Blanco, 2008), así como entender que el precio de un bien y las cantidades intercambiadas se determinan en él, a través de la interacción de estos (Blanco, 2008). En primer lugar, la oferta de un bien "recoge las intenciones de venta de los productores" (Mochón, 2009), por lo tanto, "se refiere a la relación completa entre el precio de un bien y la cantidad ofrecida del mismo" (Parkin, 2009). En ocasiones, a un grupo de oferentes se les denomina como un sector o industria (O'Kean, 2013).

Por otra parte, al analizar a los consumidores debe estudiarse su demanda, pues, aquí se ven reflejadas sus intenciones. La demanda "es la cantidad de bienes que un consumidor está dispuesto a comprar a un determinado precio en un tiempo dado" (Astudillo, 2012). La ley de la demanda determina que existe una relación inversa entre el precio y la cantidad; es decir, cuando el precio de un bien normal aumenta, su demanda disminuye (Jehle \& Reny, 2011).

Existen otras variables que influyen en la demanda como "el precio de otros bienes, la renta, los gustos y el tamaño del mercado" (Blanco, 2008). Estas variables pueden estar expresadas en la función de demanda, que es "la relación matemática que recoge la relación entre la cantidad demandada de un bien, su precio y las demás variables" (Mochón, 2009). La conducta de los consumidores se resume, entonces, en el grado en que las decisiones de consumo responden a cambios en los precios y en los ingresos (Hirshleifer, 1980). 
En primer lugar, el precio es la relación a la que se intercambian dos bienes que pueden ser absoluto (expresado en unidades de dinero) o relativo (expresado en unidades de otro bien) (Jehle \& Reny, 2011). Dentro del análisis de los precios, un indicador importante es el Índice de Precios al Consumidor (IPC), "que mide el ritmo al que los precios de los bienes y servicios de consumo cambian de un periodo a otro" (International Labor Organization / International Monetary Fund / Organisation for Economic Cooperation and Development / The Statistical Office of the European Union / The United Nations / The World Bank, 2006). La variación experimentada por este índice en un periodo de tiempo es considerado la tasa de inflación (Mochón, 2009). Por otra parte, la renta personal disponible es la remuneración pagada a los factores de producción realmente percibida por las personas, sin los impuestos directos y está disponible para el consumo y el ahorro, sabiendo que el consumo se refiere al gasto realizado por las familias en bienes y servicios (Mochón, 2009).

Ante cambios en la renta (ceteris paribus), los consumidores alteran su demanda de un bien. La teoría distingue dos tipos de bienes, normales e inferiores. En el caso de los bienes normales, un aumento de la renta (ceteris paribus), implicaría un incremento de la demanda del bien. Para los bienes inferiores, en cambio, un incremento de la renta (ceteris paribus) implica una disminución de la demanda del bien; esto se debe los bienes son sustituidos por unos de mayor calidad o mayor prestigio al incrementar el nivel de renta (Blanco, 2008). A priori se espera que el banano sea un bien normal. 
El precio de otros bienes puede afectar la

demanda se clasifican en tres: bienes independientes, complementarios o sustitutos. A continuación, los conceptos según Blanco (2008):

- Independientes: Cuando no existe una relación entre los dos bienes; esto puede observarse, debido a que una variación del precio de uno de ellos no afecta a la cantidad demandada del otro.

- Complementarios: Son bienes que se utilizan conjuntamente para cubrir una determinada necesidad. Si aumenta el precio de un bien, su cantidad demanda disminuiría y, por lo tanto, también disminuiría la demanda del bien complementario; es decir, el precio del bien complementario presenta una relación inversa.

- Sustituto: Son bienes que satisfacen por separado una misma necesidad. Cuando el precio de este bien aumenta, su cantidad demandada disminuye $y$, por lo tanto, aumenta la demanda del otro bien. En la función de demanda presenta una relación directa.

Los gustos de los consumidores presentan una relación directa con la demanda del bien; sin embargo, es una variable que no puede ser medida con facilidad. Así mismo, el tamaño del mercado que se refiere al número de consumidores presentes en él, tiene una relación directa con la demanda.

Es importante considerar que, si bien, la demanda puede ser establecida en valores absolutos, también se puede medir la sensibilidad ante cambios en sus variables; para ello, se utilizan las distintas elasticidades. La elasticidad "es una medida de la relación, en la cual, los cambios tanto del numerador como del denominador son expresados en términos porcentuales" (Hirshleifer, 1980). Por lo tanto, "la elasticidad mide qué tanto reaccionan los compradores a cambios en las condiciones del mercado, lo que permite la discusión no solo la dirección del efecto sino también su magnitud" (Mankiw, 2012). 
La elasticidad precio de la demanda mide la "variación porcentual de la cantidad demandada cuando el precio de ese bien varía en 1\%" (ceteris paribus) (Blanco, 2008). Una demanda es menos sensible al precio si esta tiene una elasticidad más baja. Blanco (2008), determina que una demanda es elástica cuando es superior a uno e implica que la variación de la cantidad demandada es porcentualmente superior a la del precio. Al contrario, si es menor a uno es inelástica e indica que la variación en la cantidad demandada es porcentualmente inferior a la del precio. Por último, si es igual a uno es una elasticidad unitaria, la variación del precio y la cantidad son proporcionalmente iguales.

Cabe mencionar que los bienes que los consumidores consideran como imprescindibles, como los alimentos, presentan una demanda más inelástica. Así mismo, los bienes con más sustitutos tienden a ser más elásticos, "una consecuencia de esta relación entre elasticidad de la demanda y posibilidad de sustitución es que los bienes más genéricos tienen demandas más inelásticas que los bienes más concretos" (Blanco, 2008).

Por otra parte, la elasticidad renta mide la "variación porcentual de la demanda cuando la renta varía en 1\%" (ceteris paribus) (Blanco, 2008), si esta es positiva se considera que es un bien normal y si es negativa un bien inferior. Además de que si está entre 0 y 1 se considera un bien de primera necesidad, que es poco sensible a variaciones; mientras que, si es mayor a 1 se considera un bien de lujo, pues, su demanda es mucho más sensible a cambios en la renta.

Por último, la elasticidad cruzada mide la variación porcentual de la demanda del bien cuando el precio de otros bienes cambia en 1\% (ceteris paribus). Si esta es positiva son sustitutos, si es negativa son complementarios y si es cero son independientes (Hirshleifer, 1980). 


\section{Métodos}

Se realizará una estimación econométrica de la demanda, a fin de analizar sus elasticidades.

La población del estudio, Estados Unidos, se considerará dentro de la muestra el periodo 2001-2016, con datos de corte mensual, lo que implica que existen 192 datos dentro de la serie. Se estudiarán datos de fuentes secundarias como:

- The United States Census Bureau.

- The United States International Trade Commission (USITC).

- The United States Bureau of Labor Statistics (BLS).

- The United States Bureau of Economic Analysis (BEA).

Después de probar diferentes formas funcionales, hasta llegar al mejor ajuste de la demanda de consumo interno de banano en Estados Unidos, se concluyó que el modelo de doble logaritmo, fue el de mejor ajuste, además de que proporciona la estimación de las elasticidades constantes en la demanda. A priori, el modelo a se expresa de la siguiente manera:

Ecuación No. 1 Especificación de la demanda interna de banano

$$
\log Q_{\text {toralt }}=\beta_{0^{-}}
$$

$\beta_{1}$ LogIPC $_{\text {Banamo }}+\beta_{2}$ LogIngreso $_{\text {per cápitat }}+\beta_{3} \log Q_{\text {rotal } t-1}+\beta_{d} \operatorname{LogIPC}_{\text {Manzana }_{t}}+e_{t}$ 
Donde la variable dependiente es $L o g Q_{t_{t o t a l}}$ que indica el logaritmo de la cantidad total importada en kilogramos de banano fresco por Estados Unidos desde el resto del Mundo, $\beta_{\mathrm{o}}$ es el intercepto de la regresión, $\beta_{1}$ es la elasticidad precio de la demanda; es decir, el coeficiente del logaritmo del índice de precios al consumidor de banano ajustado, que mide el cambio en los precios del banano a nivel del consumidor final. Por su parte, $\beta_{2}$ es el coeficiente del logaritmo del ingreso per cápita, que indica la elasticidad renta; $\beta_{3}$ es el coeficiente del logaritmo de la cantidad demandada/importada (o variable dependiente) rezagado un periodo, $\beta_{4}$ es la elasticidad cruzada que mide la relación de la manzana y el banano por medio del logaritmo del índice de precios al consumidor de manzana ajustado y por último, $e_{t}$ es el término de error de la estimación econométrica.

Para la estimación, los datos de cantidad de banano, provienen de The United States International Trade Commission (2018). Se utiliza la cuenta importaciones para consumo, puesto que, el país no es productor de banano. Se consideran los códigos arancelarios representativos de banano fresco, a base del Harmonized Tariff System. Entre 2001 y 2011, 0803002020, en 2012, 0803900020 y desde 2013 en adelante se consideran 0803900025 como certificados orgánicos y 0803900035 como frescos ${ }^{15}$.

Por su parte, se utiliza el índice de precios al consumidor del banano y la manzana ajustados mensualmente, provenientes de The United States Bureau of Labor Statistics (2018). Utilizar los índices de precios ajustados permite analizar el cambio de los precios a corto plazo, pues, elimina el efecto estacional; es decir, lo que ocurre alrededor de la misma temporada cada año y en la misma magnitud, como lo provocado por los cambios climáticos, ciclos de producción, feriados, etc.

La renta total de los consumidores estadounidenses es representada por la cuenta de ingreso personal disponible, proporcionada por el Bureau of Economic Analysis. Según esta institución, el ingreso personal es el ingreso recibido por todas las personas, por medio de todas las fuentes, como trabajo, tierras y capital, además de transferencias de

15 El cambio en los códigos se debe a cambios de nomenclaturas, mas no a cambios en el producto; sin embargo, desde 2013 el cambio implica que se considere la categoría de otros, fresco y orgánico. 
empresas y del gobierno; incluye ingresos de fuentes nacionales y extranjeras. El ingreso personal disponible es igual al ingreso personal menos los impuestos personales corrientes; es decir, es la renta realmente disponible para el gasto, inversión o ahorro de las personas (BEA, 2016).

Para el análisis y la estimación se utiliza el ingreso disponible per cápita mensual en dólares corrientes, utilizando la población residente del país obtenida de United States Census Bureau (2011, 2017). Además, debe considerarse que las funciones de demanda suelen ser auto regresivas; es decir, considerar la variable de la cantidad demandada/importada rezagada un periodo, puesto que, esta influye en la cantidad demandada/importada de la siguiente compra.

Los signos esperados son: negativo para $\beta_{1^{\prime}}$ pues, debe cumplir con la ley de la demanda, positivo para $\beta_{2^{\prime}}$ pues, se espera que el banano sea un bien normal y positivo para $\beta_{4^{\prime}}$ pues, se espera que el banano y la manzana tengan una relación sustituta, como se estableció en la teoría.

Finalmente, no se considera el tamaño del mercado como una variable, puesto que, genera problemas econométricos en la estimación, al existir la variable del ingreso per cápita que requiere del tamaño del mercado para ser estimada.

Para la estimación de modelos de demanda, existen diversos métodos, como el Almost Ideal Demand System (AIDS), el modelo Rotterdam, las regresiones Cuantílicas y los Mínimos Cuadrados Ordinarios (MCO), utilizados por varios autores que se analizaran posteriormente. $\mathrm{Si}$ bien, las primeras tres técnicas mencionadas pueden brindar mayor información desagregada, se ha considerado adecuado, para este estudio, utilizar la técnica de MCO para series de tiempo, ya que es una técnica consistente y proporciona estimadores robustos, considerados como los mejores estimadores lineales insesgados. Con esta técnica, los parámetros estimados miden el cambio absoluto o porcentual que tendrá la variable dependiente dadas las variaciones en los regresores especificados como relevantes dentro del modelo econométrico.

Las distintas variables deben ser probadas para determinar su validez estadística buscando, a su vez, que cumplan con los supuestos de 
estacionariedad. Es importante mencionar que una variable de serie de tiempo es estacionaria si su media y su varianza son constantes en el tiempo. Este supuesto es necesario para la estimación de modelos, ya que, si este no se cumple, no se puede generalizar el comportamiento de una serie de tiempo para otros periodos. Además, la estacionariedad de las variables es un principio fundamental para evitar las regresiones espurias que muestran una correlación entre las variables de series de tiempo no estacionarias de manera no correcta, por lo que son denominadas regresiones sin sentido (Gujarati \& Porter, Econometría, 2010).

Probar que las variables sean estacionarias, por lo tanto, es de alta relevancia para la estimación de los modelos; esto puede ser realizado a través de las pruebas de raíz unitaria de Dickey-Fuller Aumentada (DFA). Es sustancial notar que se utiliza la prueba aumentada, debido a que esta considera rezagos para evitar problemas de autocorrelación en la estimación que sesguen los resultados de las raíces unitarias (Mahadeva \& Robinson, 2004).

Esta prueba utiliza el estadístico $\operatorname{tau}^{16}$ y presenta como hipótesis nula que la serie cuenta con una raíz unitaria; es decir, no es estacionaria, por lo que al rechazar la hipótesis nula se puede concluir que la serie de tiempo es estacionaria. Un proceso es estacionario en diferencias cuando, una serie de tiempo que tiene una raíz unitaria, es estacionaria en primeras diferencias (Gujarati \& Porter, Econometría, 2010). Sin embargo, la metodología de los mínimos cuadrados ordinarios, puede seguirse usando como tal, con series no estacionarias, cuando al estimar la regresión sus residuos se cointegran y estos modelos son significativos; es decir, no son regresiones espurias.

La cointegración significa que una o más combinaciones lineales de las variables son estacionarias, aun cuando individualmente cada una no lo es, lo que económicamente implica que están relacionadas la una con la otra a largo plazo (Dickey, Jansen, \& Thornton, 1991). Debe, por lo tanto, comprobarse que los residuos sean estacionarios y para esto existen varias pruebas. Es común aplicar la misma prueba de Dickey-Fuller Aumentada (DFA), no obstante, al ser una prueba de cointegración esta toma el nombre de prueba de Engle -Granger Aumentada, ya que los valores críticos de la prueba DFA ya no son los apropiados (Gujarati \& Porter, Econometría, 2010).

16 Medida de correlación no paramétrica. 
Es importante notar lo mencionado por Dickey, Jansen, \& Thornton (1991) en su artículo A Primer in Cointegration with an Application to Money and Income, si bien las pruebas de cointegración y las de raíces unitarias son similares, las pruebas de raíces unitarias se realizan en series univariadas, mientras que la cointegración trata con la relación de un grupo de variables, donde cada una tiene una raíz unitaria.

Por su parte Mackinnon (2010), haciendo referencia a las investigaciones de Engle y Granger (1987), Dickey-Fuller (1979), Engle y Yoo (1987) entre otros, estima los valores críticos para las pruebas de cointegración de Engle-Granger y Dickey-Fuller para cualquier tamaño de muestra, a diferencia de los autores previamente mencionados, quienes aportaban tablas para pocos tamaños de muestras. Gracias a este aporte, es posible encontrar el valor crítico correcto para la estimación de cada modelo para distintos niveles de confianza.

A continuación, se presenta la forma de cálculo. Es importante notar que cada Beta es presentado por Mackinnon, a base del número de variables independientes $(\mathrm{N})$, nivel de significancia y parámetros considerados en la prueba; sabiendo que $T$, hace referencia al número de observaciones consideradas, la forma de cálculo es la siguiente:

$$
B_{\infty}+\frac{B_{1}}{T}+\frac{B_{2}}{T^{2}}
$$

Esta fórmula es usada en los modelos para la contrastación de la cointegración.

Por otra parte, se realizarán pruebas para determinar que el modelo cumpla con los supuestos de los mínimos cuadrados ordinarios, según lo establecido por Gujarati y Porter (2010). Para ello, se probará que no exista multicolinealidad; es decir, que las variables explicativas no estén correlacionadas entre sí y sea posible aislar sus efectos individuales sobre la variable dependiente.

Además, se realizarán pruebas para que no exista presencia de heteroscedasticidad, que se da cuando la varianza del término error no 
es constante para todos los valores de las variables independientes $y$, por lo tanto, genera problemas en la estimación, haciendo que los estimadores sean ineficientes ${ }^{17}$, pues, perderían su característica de varianza mínima que podría ser sesgada.

De igual manera, se estudia la presencia de autocorrelación que significa que el término error de un periodo está correlacionado con el término de error de cualquier otro periodo, ya que este problema es frecuente en series de tiempo. Este problema lleva, de igual manera, a estimadores ineficientes y errores estándares sesgados que pueden implicar contrastaciones estadísticas incorrectas o intervalos de confianza sesgados.

Dada la existencia de alguno de estos problemas, se realizarán las correcciones necesarias. Además, debe recalcarse que se estudiará también que las variables cumplan con las relaciones que indica la teoría, presentada previamente. Para la contrastación de las hipótesis se ha establecido el nivel de significancia $(\alpha)$ del $5 \%$; para la estimación del modelo se utilizará el software econométrico de Eviews 8 y para otras estadísticas el software de Excel 2011.

17 A pesar de ser ineficientes, los estimadores siguen siendo insesgados. 


\title{
Resultados
}

\author{
Previo a la estimación, los datos fueron probados para \\ determinar si estos eran estacionarios; se concluyó que, \\ a pesar de que algunas variables no lo eran, el modelo \\ se cointegra, haciendo que sea posible la estimación.
}

El modelo especificado fue probado, sin embargo, se concluyó óptimo eliminar la variable del precio de la manzana ya que no era estadísticamente significativa y su presencia en el modelo generaba problemas como heteroscedasticidad y multicolinealidad. Siguiendo con la metodología planteada, se realizaron varias pruebas para determinar si la variable debía ser eliminada, como una matriz de correlación que mostró un nivel de correlación alto entre el IPC de la manzana y el ingreso per cápita; además, se realizó la prueba del factor de inflación de la varianza (VIF) y se determinó que el IPC de la manzana tiene un valor mayor a 10, por lo tanto, hay multicolinealidad. Adicionalmente, para tomar la decisión de retirarla, se analizó el aporte que tiene cada variable al modelo por medio del coeficiente de Theil (m), llegando a la conclusión de que la variable que menos aporta a la explicación fue la representante del precio de la manzana. Si bien esta variable fue eliminada del modelo, cabe mencionar que presentó un signo positivo; es decir, una relación directa indicando que la manzana podría ser considerada un bien sustituto del banano, sin embargo, este no fue significativo. Por lo que el modelo de la demanda interna de banano en Estados Unidos es:

\section{Ecuación No. 2 Demanda interna de banano}

$$
\log Q_{\text {total }_{t}}=9.1825-0.2259{\log I P C_{\text {Banano }_{t}}}+0.4074 \text { LogIngreso }_{\text {per cápita }_{t}}+0.381 \log Q_{\text {total }_{t-1}}
$$

A continuación, se presenta la tabla con los resultados de la regresión utilizados para la contrastación de hipótesis. 
Tabla No. 1 Resultados de la estimación de demanda interna de banano

\begin{tabular}{|c|c|}
\hline Periodo & $\begin{array}{l}2001-2016 \\
\text { Log Q total }\end{array}$ \\
\hline Log IPC Banano Ajustado & -0.2459 \\
\hline Prob. & 0.0559 \\
\hline Log Ingreso Per Cápita & 0.4074 \\
\hline Prob. & 0.0000 \\
\hline $\log O(t-1)$ & 0.3810 \\
\hline Prob. & 0.0000 \\
\hline C & 9.1825 \\
\hline Prob. & 0.0000 \\
\hline R cuadrado & 0.3961 \\
\hline R cuadrado ajustado & 0.3864 \\
\hline Valor F & 40.8899 \\
\hline Prob. F & 0.0000 \\
\hline
\end{tabular}

Fuente: Elaborado por la autora.

La estimación indica que el logaritmo del IPC del banano, el logaritmo del ingreso per cápita y el logaritmo de la cantidad rezagada un periodo explica en conjunto $38.6 \%$ a la demanda de banano en Estados Unidos. Adicionalmente, el modelo ha determinado tanto la elasticidad precio, como la elasticidad renta, pues, indica que, si el IPC del banano aumenta $1 \%$, la cantidad demandada de banano disminuirá en $0.246 \%$ (o viceversa); claramente al mostrar una relación inversa entre el precio y la cantidad se cumple lo indicado en la teoría de la ley de la demanda. Además, al tener un valor menor a uno, se concluye que el banano es un bien inelástico en el mercado estadounidense; es decir, que la variación de la cantidad es menos sensible a la variación del precio. 
En cuanto a la renta, si el ingreso per cápita de los americanos se incrementa en $1 \%$, la demanda de banano aumentará en $0.4074 \%$, mostrando una relación directa entre estas variables, como establece la teoría. Al ser un valor positivo y encontrarse entre cero y uno, indica que el banano es un bien normal y necesario para los consumidores estadounidenses.

Por último, se determinó que la demanda de banano del periodo está influenciada por la demanda de su periodo precedente de manera positiva; es decir, mientras más grande fue la demanda de banano en el periodo anterior, mayor será la demanda en este periodo. Esto puede estar relacionado a los gustos y preferencias de los consumidores quienes indican que, mientras más les guste a los consumidores esta fruta, más comprarán en el siguiente periodo. 


\section{Discusión}

Los distintos estudios de estimación de demanda utilizan diferentes metodologías para la estimación de sus modelos; varios de ellos prueban los mejores ajusten con modelos AIDS, Rotterdam, lineal o doble logarítmico, como se mencionó anteriormente. La utilización y el buen ajuste que brinda el modelo logarítmico en varias investigaciones, corroboran la decisión de elegir esta forma funcional en la estimación, sin importar qué forma funcional estimen, las investigaciones buscan llegar a las elasticidades de los bienes. A continuación, se analizarán varios estudios relacionados en cuanto a sus metodologías y sus resultados.

En cuanto a la metodología, Paudel, Adhikari, Houston y Paudel (2010) en su investigación sobre la demanda de frutas en Estados Unidos periodo 1980 - 2003 analiza varias formas funcionales y compara favorablemente al modelo logarítmico con el AIDS, lo que corrobora la decisión de implementar esta metodología.

Adicionalmente, un estudio, que analiza la demanda de frutas en Sri Lanka entre 1985 y 2010, realizado por Weerahewa, Rajapakse y Pushpakumara (2012), utiliza formas lineales y logarítmicas para su estimación de la demanda. En este, la cantidad demandada es función del precio de la fruta, el ingreso per cápita (utilizando el gasto en consumo privado como proxy), una variable de preferencia de los consumidores y la cantidad demanda rezagada un periodo, ratificando la importancia de la inclusión de esta variable al modelo. Esta investigación, para el caso de la demanda de banano, utiliza una forma lineal. 


\section{Elasticidad precio:}

Por su parte Paudel, Adhikari, Houston y Paudel (2010), concluyeron a través de distintos modelos que, el banano es un bien inelástico al igual que en la presente investigación, encontrando una elasticidad precio con el modelo logarítmico de - 0,25 similar a $-0,246$ de los resultados previamente presentados. Por otra parte, un estudio realizado por USDA en el periodo 1998-2010, al estimar la elasticidad precio de la demanda de banano para el mercado americano, determinó que este bien cuenta con una elasticidad unitaria $(-1,01)$, significativa al $10 \%$, pero, lo compara con un estudio realizado en 1993 por Huang, donde es inelástica (-0.5) similar a lo determinado en esta investigación (Okrent \& Alston, 2012).

Weerahewa, Rajapakse y Pushpakumara (2012) en su análisis en Sri Lanka concluyen que la demanda de banano responde altamente a variaciones en el precio y el ingreso, obteniendo una elasticidad precio en este país de -0.95. Por último, un estudio realizado en 2009 por Yen, Huang y Lin para el mercado estadounidense, difiere entre frutas convencionales y frutas orgánicas. Al estimar la demanda del banano convencional determinó que este es inelástico con un coeficiente de elasticidad de -0.70, diferente a la estimación de la demanda de banano orgánico que es altamente elástico (Yen, Huang , \& Lin , 2009).

Todas estas estimaciones de la elasticidad precio en la demanda de banano, indican que la elasticidad obtenida en este análisis es correcta. Sin embargo, cabe mencionar que, un estudio que analiza la demanda de banano del consumidor en Alemania por medio de la diferenciación de tipos de hogares en base a la renta, demostró que la elasticidad precio es mayor para los hogares con rentas más bajas, dado que una reducción del precio del banano implicaría un mayor incremento del bienestar (medido en dinero) para las familias de este segmento, que para las familias de los segmentos más altos (Bureell \& Henningsen, 2001). 


\section{Elasticidad renta:}

Distintas investigaciones han concluido que en el mercado americano el banano es un bien normal necesario por medio de su estimación de la elasticidad renta, al igual que los resultados de esta investigación. En primer lugar, Paudel, Adhikari, Houston y Paudel (2010) utilizando la elasticidad gasto (como proxy de la renta) determinó que es un bien normal y necesario en los modelos logarítmicos y Rotterdam (0.73 y 0.64) utilizando una significancia del $10 \%$.

De igual manera, un estudio realizado por Okrent y Alston para USDA (2012) utilizando a su vez la elasticidad gasto, obtuvo un coeficiente de 0.05, confirmando lo antes indicado; es decir, que el banano es un bien necesario normal. Si bien los coeficientes varían de estudio en estudio, las variaciones no son amplias y llevan a las mismas conclusiones.

No obstante, para el caso de Sri Lanka, Weerahewa, Rajapakse y Pushpakumara (2012) determinaron que la elasticidad renta en este país es de 1.04, que indica que el banano es considerado un bien normal de lujo. Concluyeron que en ese mercado de frutas el consumo es más afectado por cambios en el ingreso que por cambios en los precios al igual que ocurre en el mercado americano; mientras que, Bureell \& Henningsen (2001) en Alemania determinan que el banano es un bien normal.

\section{Elasticidad cruzada:}

El mayor debate se encuentra al analizar la relación que existe entre el banano y la manzana, como se menciona previamente. La estimación indicó que estos podrían ser bienes sustitutos; sin embargo, al no haber sido significativo implica aún un mayor análisis. Por una parte, Paudel, Adhikari, Houston y Paudel (2010) al estimar la elasticidad cruzada en la demanda de banano (AIDS, LOG) encontraron que la manzana presentó relaciones complementarias; mientras que, al estimar la demanda de manzana (AIDS), el banano presentó una relación sustituta. 
Por otro lado, Okernt y Alston (2012) al utilizar un modelo GODDS para la demanda de banano en el periodo de 1998-2010, determinaron que la manzana presentó signo negativo, lo que indica que serían bienes complementarios y, así mismo, al estimar la demanda de manzanas, el banano también fue considerado complementario; no obstante, ninguna de estas variables fue significativa ni al 10\% de significancia.

Por su parte, Yen, Huang y Lin (2009) realizaron un estudio diferente en el mercado americano, al distinguir entre frutas convencionales y frutas orgánicas. Los resultados obtenidos del análisis de elasticidad cruzada mostraron que la manzana y el banano convencional presentan una relación complementaria (-0.08); mientras que, al estimar la demanda de banano orgánico, determina que este es sustituto del banano convencional y sustituto de la manzana orgánica. El estudio afirma que los consumidores tienden a incrementar sus compras de fruta orgánica cuando sube el precio de las frutas convencionales. Así mismo determina que los consumidores tienden en mayor magnitud a sustituir fruta orgánica por fruta convencional, que viceversa.

Sin embargo, el estudio que más aporta a la comprensión de esta relación es el de Bureell y Henningsen en Alemania (2001), estos autores al distinguir el nivel de renta de los consumidores en tres tipos de hogares permitieron analizar la relación de estos bienes bajo distintas circunstancias. Así, el estudio determinó que la manzana y el banano son claros sustitutos para el hogar con menores ingresos, pero, no para los dos otros hogares con ingresos más altos. Cabe recalcar que esta relación se presentó tanto al estimar la demanda de banano, como al estimar la demanda de manzana. Además, determinó que estas dos frutas son sustitutas para el resto de frutas en los hogares de mayores niveles de ingresos (Bureell \& Henningsen, 2001).

Por consiguiente, si bien el presente estudio encontró una relación positiva, es decir, sustituta entre los bienes, esto puede deberse a que se ha considerado un solo tipo de consumidor (un solo nivel de ingreso), mientras que como se ve en el estudio de Bureell \& Henningsen (2001) para Alemania, el nivel de ingreso de la familia influye a la relación de estos bienes. Pues, para familias con ingresos menores, la manzana y el banano son sustitutos notorios; sin embargo, familias con ingresos más altos pueden optar por adquirir ambos bienes, es decir, complementarios. 
A modo de conclusión, se puede decir que, después de analizar la demanda de banano en Estados Unidos, se encuentra relevante que los proveedores mundiales de banano consideren las características del principal mercado de importación de este bien. En primer lugar, que el precio, la renta y la cantidad rezagada un periodo explican su demanda en un $38.6 \%$ en Estados Unidos. También, se concluyó que el banano presenta una demanda inelástica al precio de -0.246 , coeficiente similar al obtenido por otros autores.

Además, se determinó, que en este mercado el banano es considerado un bien normal y necesario debido a su relación con el ingreso per cápita que es poco sensible a variaciones del ingreso medio de los consumidores estadounidenses, ya que su elasticidad renta en el periodo es de 0.4074, corroborando la relación indicada en la teoría, puesto que, no es un bien inferior. A su vez, se comprobó que la demanda de este bien está influenciada por su demanda precedente, variable también usada por otros autores.

No obstante, a pesar de no ser significativo, la elasticidad cruzada entre la manzana y el banano generó un mayor debate, puesto que, se encontró una relación sustituta entre los bienes; mientras que, otras investigaciones encontraron relaciones complementarias, destacando que puede deberse a que solo se consideró un tipo de consumidor en la investigación y no consumidores con distintos niveles de renta, para quienes estas frutas puedan implicar distintas decisiones, por lo que este tema podría ser sujeto de estudio de una nueva investigación. 


\section{Bibliografía}

Astudillo, M. (2012). Fundamentos de Economía. México: Instituto de Investigaciones Económicas: Probooks.

Blanco, J. (2008). Economía, Teoría y Práctica (Quinta edición ed.). Madrid, España: McGraw-Hill.

Bureau of Labor Statistics, BLS. (2018). CPI-All Urban Consumers, CPI-Average Price Data. Recuperado el 21 de Marzo de 2018, de Bureau of Labor Statistics: https://beta.us.gov/ dataQuery/find?st $=0 \& r=20 \&$ more $=0 \& q=$ bananas + cpi

Bureell, A., \& Henningsen, A. (2001). An empirical investigation of the demand for bananas in Germany. Recuperado el 24 de Septiembre de 2018, de Agrarwirtschaft 50 (2001), Heft 4: https-//ageconsearch.umn.edu/bitstream/98885/2/3_Burrell.pdf

Dickey, D., Jansen, D., \& Thornton, D. (Marzo-Abril de 1991). A Primer On Cointegration with an Application to Money and Income. Obtenido de Federal Reserve Bank of St. Louis: https://files.stlouisfed.org/files/htdocs/publications/review/91/03/Primer_Mar_ Apr1991.pdf

Food and Agriculture Organization, FAO. (2017). Situación del mercado del Banano. Recuperado el 01 de Mayo de 2018, de Organización de las Naciones Unidas para la Alimentación y la Agricultura: http://www.fao.org/3/a-i7410s.pdf

Food and Agriculture Organization, FAO. (2018). FAOSTAT - Bananas. Recuperado el 22 de Marzo de 2018, de Organización de las Naciones Unidas para la Alimentación y la Agricultura: http://www.fao.org/faostat/en/\#data/OC

Gujarati, D. N., \& Porter, D. C. (2010). Econometría. México: Mc Graw Hill.

Hirshleifer, J. (1980). Teoría de precios y sus aplicaciones (Primera edición ed.). (INCAE, Ed.) México: Prentice-Hall Hispanoamericana.

International Institute for Sustainable Development, IISD. (2014). The State of Sustainability Initiatives Review 2014 Standards and the Green Economy. Recuperado el 23 de Agosto de 2018, de https://www.iisd.org/sites/default/files/pdf/2014/ssi_2014.pdf\#page $=97$

International Labor Organization / International Monetary Fund / Organisation for Economic Cooperation and Development / The Statistical Office of the European Union / The 
United Nations / The World Bank. (2006). Manual del índice de precios al consumidor: Teoría y práctica. Recuperado el 10 de Mayo de 2018, de Fondo Monetario Internacional: https://www.imf.org/external/pubs/ft/cpi/manual/2004/esl/cpi_sp.pdf

Jehle, G., \& Reny, P. (2011). Advance Microeconomic Theory (Tercera ed.). Essex: PEARSON. MacKinnon , J. (Enero de 2010). Critical Values for Cointegration Tests. Recuperado el 01 de Noviembre de 2018, de Queen's Economics Department: http://qed.econ.queensu. ca/working_papers/papers/qed_wp_1227.pdf

Mahadeva , L., \& Robinson, P. (Julio de 2004). Prueba de raíz unitaria para ayudar a la construcción de un modelo. Recuperado el 04 de Noviembre de 2018, de Centro de Estudios Monetarios Latinoamericanos, Asociación Regional de Bancos Centrales, Banco De Inglaterra.: http://www.cemla.org/PDF/ensayos/pub-en-76.pdf

Mankiw, G. (2012). Principios de Economía (6ta Edición ed.). México: Cengage Learning.

Mochón, F. (2009). Economía, Teoría y Política (Sexta Edición ed.). Madrid, España: McGraw-Hill.

O’Kean, J. (2013). Economía. Madrid: McGraw Hill.

Okrent, A. M., \& Alston, J. M. (Agosto de 2012). The Demand for Disaggregated Food- AwayFrom-Home and Food-at-Home Products in the United States. Recuperado el Agosto de 2018, de Journal of International Food \& Agribusiness Marketing: https-//www.ers. usda.gov/webdocs/publications/45003/30438_err139.pdf?v=41148.pdf

Parkin, M. (2009). Economía (Octava Edición ed.). México: Pearson Education.

Paudel, L., Adhikari, M., Houston, J., \& Paudel, K. P. (2010). Low carbohydrate information, consumer health preferences and market demand of fruits in the United States. Recuperado el 16 de Abril de 2018, de Applied Economics Letters: https://www.scopus.com/record/display.uri?eid=2-s2.0-76749106931\&origin=resultslist\&sort=plf-f\&s$\mathrm{rc}=\mathrm{s} \& \mathrm{st} 1=$ Estimation + of + demand + banana

Smith, A. (1776). Investigación sobre la naturaleza y causas de la riqueza de las naciones. Londres.

The Packer. (2013). Fresh Trends. Recuperado el 11 de Septiembre de 2018, de A retail transformation: http://mydigimag.rrd.com/publication/?i=152012\#\{\%22issue_ id\%22:152012,\%22page\%22:8\} 
The Packer. (2016). Commodity: Bananas. Recuperado el 25 de Agosto de 2018, de Produce Market Guide, a product of The Packer: https://www.producemarketguide.com/produce/bananas\#nutrition

The Packer. (2016). Fresh Trends - Bananas. Recuperado el Julio de 2018, de Produce Market Guide: https://www.producemarketguide.com/sites/default/files/fresh\%20trend\%20 files.tar/fresh\%20trend\%20files/bananas_fresh-trends.pdf

United Nations Conference on Trade and Development, UNCTAD. (2015). Banano Perfil de INFOCOMM. Recuperado el 09 de Agosto de 2018, de UNCTAD: http-//unctad.org/ es/PublicationsLibrary/INFOCOMM_cp01_Banana_es.pdf

United States Bureau of Economic Analysis, BEA. (Octubre de 2016). Glossary: National Income and Product Accounts. Recuperado el 01 de Julio de 2018, de United States Bureau of Economic Analysis: www.bea.gov/national/pdf/glossary.pdf

United States Bureau of Economic Analysis, BEA. (29 de Marzo de 2018). 2.6 Personal Income and Its Disposition, Monthly. Recuperado el Junio de 2018, de National Income and Product Accounts: https://apps.bea.gov/iTable/iTable.cfm?reqid=19\&step $=2 \#$ reqid $=19 \&$ step $=3 \&$ isuri $=1 \& 1921=$ survey $\& 1903=58$

United States Census Bureau. (Septiembre de 2011). Monthly Intercensal Resident Population Estimates for the United States: April 1, 2000 to July 1, 2010. Recuperado el Mayo de 2018, de Population Division: https://www.census.gov/data/datasets/time-series/ demo/popest/intercensal-2000-2010-national.html

United States Census Bureau. (Diciembre de 2017). Table 1. Monthly Population Estimates for the United States: April 1, 2010 to December 1, 2018. Recuperado el Mayo de 2018, de Population Division: https://www.census.gov/data/tables/2017/demo/popest/nation-total.html

United States Department of Agriculture, USDA. (2016). Table 7-Food expenditures by families and individuals as a share of disposable personal income. Recuperado el 09 de Agosto de 2018, de Food Expenditure Series: https://www.ers.usda.gov/data-products/food-expenditure-series/food-expenditure-series/\#Archived\%20Food\%20Expenditure\%20Tables

United States Department of Agriculture, USDA. (26 de Julio de 2017). Loss-Adjusted Food Availability-Fruits. Recuperado el 27 de Marzo de 2018, de Food Availability (Per Capita) Data System: https://www.ers.usda.gov/data-products/food-availability-per-capita-data-system/ 
United States International Trade Comission, USITC. (2018). U.S. Imports For Consumption. Recuperado el 16 de Abril de 2018, de Data Web USITC: https://dataweb.usitc.gov/ scripts/INTRO.asp

Weerahewa, J., Rajapakse, C., \& Pushpakumara, G. (02 de Octubre de 2012). An analysis of consumer demand for fruits in Sri Lanka. 1981-2010. Recuperado el 16 de Abril de 2018, de Appetite- ELSEVIER: https://www.scopus.com/ record/display.uri?eid=2-s2.0-84877304796\&origin=resultslist\&sort=plf-f\&s$\mathrm{rc}=\mathrm{s} \& \mathrm{st} 1=$ Estimation $+\mathrm{of}+$ demand + banana\&st $2=$

Yen, S., Huang , C., \& Lin , B.-H. (Agosto de 2009). U.S. Demand for Organic and Conventional Fresh Fruits: The Roles of Income and Price. Recuperado el Septiembre de 2018, de Sustainability: https-//pdfs.semanticscholar.org/5cc1/3a1dc7eee594ceeb8e30eb137088a96ad45d.pdf

Zeballos, E., \& Anekwe, T. (Abril de 2018). The Association Between Nutrition Information Use and the Healthfulness of Food Acquisitions. Recuperado el 08 de Agosto de 2018, de United States Department of Agriculture: https://www.ers.usda.gov/webdocs/publications/88531/err-247.pdf?v=43209 